\section{Statistics Flaws}

TO THE EDITOR: It was fantastic to participate in the training run for the new JNMT Twitter-based journal club (@JNMTClub using \#JNMTClub). Although only a small number of invited participants were engaged, it promises to be a fantastic medium for nuclear medicine technologists to connect, engage, learn, and participate in continuing education among a global community of colleagues. The format will be of benefit to those experienced in social media and the novice. The JNMT article discussed during the Twitter-based journal club was Dako et al. (1), examining the patients' perception of quality at the point of care in PET. The Twitter discussion asked the question "Do you think the results of the survey are meaningful?"

The results lack any statistical validity in my opinion. The Likert scale used rendered the data ordinal in nature. The statistical analysis treated the data as though it were continuous data, and this represents a fatal flaw. Ordinal data should not be represented as a mean; the median is the appropriate expression for central tendency (2). Indeed, had the median been cited, it may have given a better insight into any trends. This flaw is easily rectified with appropriate statistical treatment; however, the mean data and associated $P$ values are inappropriate and leave no conclusions that can be reliably drawn. This statistical flaw is not uncommon in the medical literature but represents reputation damage to the research itself, the review process of the journal, the journal, and the broader profession. A working understanding of appropriate use and meaning of common statistical tools is essential for all.

\section{REFERENCES}

1. Dako F, Wray R, Awan O, Subramaniam RM. Adapting a standardized, industryproven tool to measure patients' perceptions of quality at the point of care in a PET/CT center. J Nucl Med Technol. 2017;45:285-289.

2. Currie G, McCruaig C, Di Prospero L. Systematically reviewing a journal manuscript: a guide for reviewers. J Med Imaging Radiat Sci. 2016;47:129-138.

\section{Geoff Currie \\ Charles Sturt University P.O. Box U102, CSU \\ Wagga Wagga, NS 2650, Australia \\ E-mail: gcurrie@csu.edu.au}

Published online May 3, 2018

DOI: $10.2967 /$ jnmt.118.211730

\section{Use of Parametric Tests to Analyze Ordinal Data}

REPLY: We would like to respond to the critique recently submitted to the Journal of Nuclear Medicine Technology regarding our article titled "Adapting a Standardized, Industry-Proven Tool to Measure Patients' Perceptions of Quality at the Point of Care in a PET/CT Center." First, we would like to thank Dr. Geoff Currie for his insightful comments regarding a common debate in the epidemiology and public health community regarding how to handle ordinal data. In his critique, Dr. Currie writes, "The Likert scale used rendered the data ordinal in nature. The statistical analysis treated the data as though it were continuous data, and this represents a fatal flaw."

Much has been written regarding the appropriate analysis of ordinal data, and whether parametric tests such as the $t$ test can be used to treat ordinal data as continuous. For example, Dr. Geoff Norman, a renowned expert in medical education research methodology, has shown that parametric tests can be used to analyze ordinal data $(1,2)$. Moreover, he concludes that parametric tests are generally more robust than nonparametric tests when analyzing ordinal data such as that seen in Likert scales, even when statistical assumptions (such as normal distribution of data) are violated.

Thus, we disagree with Dr. Currie's critique and stand by the results of our article. We believe the $P$ values from our data in Table 3 to be valid because they represent results from a 2-sample $t$ test, a parametric test that treated ordinal data from a 7-point Likert scale as continuous. We do agree, however, with Dr. Currie that reporting median values as opposes to means may have been a better expression for central tendency, because the data from a Likert scale is ordinal. On the other hand, reporting means does not invalidate a study using a Likert scale, as eloquently pointed out by Sullivan et al. (1).

Thank you so much for allowing us to explore such an important topic regarding statistical methods in research. We hope this response is informative and sheds insight into why we chose to handle ordinal data as continuous.

\section{REFERENCES}

1. Sullivan GM, Artino AR Jr. Analyzing and interpreting data from Likert-type scales. J Grad Med Educ. 2013;5:541-542.

2. Norman G. Likert scales, levels of measurement and the "laws" of statistics. $A d v$ Health Sci Educ Theory Pract. 2010;15:625-632.

\author{
Omer Awan, MD, MPH, CIIP \\ Farouk Dako, MD, MPH* \\ *Temple University Hospital \\ 3401 North Broad St. \\ Philadelphia, PA 19140 \\ E-mail: fdako123@gmail.com
}

Published online May 3, 2018. DOI: 10.2967/jnmt.118.211805 